Ress ẽ as

\title{
Crítica y Emancipación, Revista latinoamericana de ciencias sociales, año l, núm. 1, junio 2008.
}

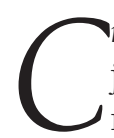
rítica y Emancipación nace a la mitad del año 2008, justo cuando los Estados Unidos y el modelo neoliberal comienzan a mostrar signos de agonía. La crisis del sector de la vivienda, que comenzó en 2007, ahora se traslada a Wall Street, a pesar de la inyección de miles de millones de dólares que el gobierno de George Bush invirtió para salvar a los grandes bancos y compañías transnacionales de origen estadounidense. Se trata de una crisis que comienza a contaminar a las economías más industrializadas del planeta y que en el corto plazo tendrá serias consecuencias para los países periféricos.

La preocupación ha llegado a tal grado que el tema de la Cumbre Iberoamericana, realizada en los últimos días de octubre de 2008 en San Salvador, El Salvador, fue justamente la crisis financiera y las medidas que debieran tomar los países para salir de ella. En este tenor, una idea que prevaleció hasta en los ánimos de los presidentes más neoliberales, es la necesidad de mayor intervención estatal, quizá como un efecto demostración de las medidas que tomó el presidente de los Estados Unidos. Pero lo que más llama la atención fue la propuesta de que España pudiera participar, en representación de los países de Iberoamérica, en la cumbre del G-20, que tendría lugar el 15 de noviembre de este año en Washington para definir el rumbo de los países industrializados, una especie de Bretton Woods en un contexto de globalización neoliberal. La exclusión de países como España en la discusión sobre la crisis entraña, indudablemente, un problema que tiene que ver con el poder y la toma de decisiones sobre asuntos que afectan a todos los países del mundo por un puñado de países ricos que imponen su voluntad sobre el resto, pero también revela que a pesar de los procesos de independencia y descolonización los gobiernos de los países iberoamericanos se asumen colonizados.

Podríamos ir más lejos al decir que la recesión de la economía estadounidense tuvo un precio político muy alto para el presidente Bush: la derrota del republicano John McCain en las elecciones para la presidencia de los Estados Unidos, frente a su rival demócrata Barack Obama. Debemos recordar que en 1992 George Bush padre perdió la reelección por una recesión económica que golpeaba a la economía doméstica y que la administración no supo resolver. El peso económico y financiero que tiene Estados Unidos en el mundo de hoy afecta a todos los países, por lo mismo, las bolsas de valores, el comercio internacional, los precios del petróleo y las remesas, entre otros, están comenzando a resentir los impactos de la recesión estadounidense, que más temprano que tarde tendrá un costo social muy grande para los países periféricos y para los propios países de 
reciente desarrollo. En el caso de España se comienza a observar un incremento importante en el desempleo.

En medio de esta crisis, sale a la luz el primer número de Crítica y Emancipación, que se suma a una serie de esfuerzos en el plano editorial y en el impulso de proyectos de investigación y docencia que abonan al gran proyecto de CLACSO: impulsar el pensamiento crítico latinoamericano.

Como bien señala Emir Sader, en una síntesis magistral que introduce este primer número de la Revista, la América Latina de hoy se encuentra en un contexto muy diferente al de los años setenta y ochenta, caracterizados por una profunda crisis económica y sociopolítica que en varios países se expresó en la instauración de atroces dictaduras militares, para hacer prevaler un modelo económico y una forma de pensar.

Hoy, sin embargo, asistimos al empobrecimiento y exclusión de amplios sectores sociales de los países de la región. La crudeza del neoliberalismo se ha ensañado con las clases más desposeídas. El pensamiento social ha estado dominado por la hegemonía de una ciencia social sin compromiso con las grandes masas de la población más vulnerable. En este contexto, Crítica y Emancipación se propone abrir el debate, fomentar la creatividad de pensamiento, la reelaboración conceptual y metodológica que sirva para entender lo que hoy está ocurriendo en el mundo y en la región, así como contribuir al cambio social, superar, como refiere Emir Sader, la crítica-crítica, para colocarse en la perspectiva de un futuro distinto para América Latina.

Este primer número de la Revista se estructura en ocho apartados: Constituyente boliviana: espacio de disputa hegemónica; el declive de la cultura francesa; cultura y política en América Latina; diálogos latinoamericanos; perspectivas; revista de nuestra América; lecturas críticas y la sección de documentos. Por supuesto, por razones de espacio no haré referencia a cada uno de ellos, sólo me limitaré a comentar y resumir algunos pues, en última instancia, se trata de invitar al público interesado a consultar la revista.
En el primer apartado podemos encontrar dos artículos de gran actualidad referidos a la cuestión boliviana: el primero, escrito por Álvaro García Linera - actual viveprecidente de Bolivia -, denominado "Empate catastrófico y punto de bifurcación". El segundo, de la autoría de Raúl Prada Alcoreza, analiza la nueva Constitución Política del Estado.

García Linera plantea que frente a la crisis que llevó a Evo Morales al poder, hoy se enfrentan dos proyectos: el neoliberal y el que se encuentra en proceso de construcción, de corte indígena y popular. Esta confrontación ha provocado una nueva crisis de hegemonía cuya resolución puede caminar en dos sentidos: a través de la insurrección o por la vía democrática. El autor describe tres etapas: 1) la crisis del Estado y su visibilización; 2) empate catastrófico y construcción de hegemonía; y 3) nueva constitución política del Estado.

En la primera refiere que en Bolivia se presentó una crisis del Estado neoliberal, caracterizada por dificultades en la correlación de fuerzas, en la estructura de fuerzas con capacidad de decisión. Esta crisis, que comenzó en el año 2000, se manifestaba en el hecho de que las ideas del bloque empresarial vinculado a los grupos de inversión extranjera, exportadores, banca y la elite política habían perdido la capacidad para poder definir las políticas públicas.

$\mathrm{El}$ autor refiere dos rasgos definitorios de la crisis: por una parte, las ideas dominantes que presentaban a la inversión extranjera como motor de la economía, a la globalización y exportación como elementos fundamentales de la modernidad, que se correspondía, en el ámbito de lo político, con la coalición de partidos que definía la gobernabilidad. Por otra parte, las instituciones habían perdido eficacia: el parlamento, expropiado por el ejecutivo, ya no era un espacio para el debate político. Asimismo, el ejecutivo estaba limitado por las empresas extranjeras.

Esta crisis estatal puede ser superada; sin embargo, de no ser posible conduciría a un "empate catastrófico", 
que es, en términos gramscianos, una etapa de crisis caracterizada por tres elementos: 1) confrontación de dos proyectos con capacidad de movilización, de atracción y seducción de fuerzas sociales; 2) confrontación en el ámbito institucional del bloque dominante y el ascendente; 3) parálisis de mando estatal y la irresolución.

La salida al "empate catastrófico" es la construcción de hegemonía marcada por la conflictividad, la cual tiene que acabar en algún momento, alcanzando de esta manera estabilidad, que para el caso boliviano puede significar la reconstrucción del neoliberalismo o bien un Estado nacional, indígena, popular, revolucionario. Es decir, se llega a un punto de bifurcación que puede resolverse de manera insurreccional o por la vía democrática. En todo caso, debe mostrarse o imponerse un liderazgo moral, de manera que si los indígenas quieren consolidarse como núcleo del Estado, tienen que mostrar que son capaces de recoger y llevar adelante los intereses de la clase media y del empresariado boliviano. En una palabra, los indígenas están obligados a hablar con los adversarios. Para el autor, la nueva constitución política del Estado deja un núcleo indígena popular e incorpora a otros sectores, incluyendo el empresariado. En otro sentido, lo que cambió con la nueva constitución y con el nuevo gobierno son las formas de hacer las cosas.

En la nueva Constitución hay puntos claves: en el ámbito de los recursos naturales, garantiza la nacionalización del gas y el petróleo que ninguna ley puede privatizar, y esto incluye la refinación, comercialización y definición de precios. La nueva Constitución establece la retroactividad de la ley en los casos de corrupción. Todos los casos son investigables: presidentes, vicepresidentes y ministros.

El artículo de Raúl Prada Alcoreza, denominado "Análisis de la nueva Constitución Política del Estado", analiza de manera detallada las características de la nueva Constitución, sancionada después de que asumió la presidencia Evo Morales. La esencia de la Constitución reside en que se da forma a la noción de Estado interventor, protector de los recursos naturales, de bienestar, que incorpora formas y prácticas de los pueblos originarios, que reconoce su derecho a la libre determinación. Pero en todo caso, se trata de una constitución que permite el tránsito hacia un Estado plurinacional, cuyo énfasis es la descentralización administrativa-política y el sistema de autonomías.

Esta constitución de transición combina los desarrollos evolutivos de los derechos, deberes y garantías liberales con demandas indígenas constitucionalizadas que dotan de marco legal al proceso de nacionalización.

El autor refiere la estructura constitucional y destaca el carácter del Estado boliviano como unitario social de derechos plurinacional, comunitario, libre, independiente, soberano, democrático, intercultural, descentralizado y con autonomías. Establece que la soberanía reside en el pueblo y se ejerce de manera directa. Recupera la dimensión de género, que junto con lo plurinacional y comunitario, están presentes como ejes transversales en todo el documento.

Los derechos fundamentales incluyen: el derecho a la vida, al agua y la alimentación, al hábitat y a la vivienda, y al acceso a los servicios básicos de agua potable, alcantarillado, electricidad, gas domiciliario, postal y telecomunicaciones.

Un aspecto central en la nueva Constitución son los pueblos indígenas a los que se atribuye posibilidades civilizatorias alternativas a los de la modernidad capitalista, lo que implica considerar a los pueblos indígenas no sólo como población y ethnos, sino también como saberes y prácticas que deberán ser recuperadas en la perspectiva de un mundo sustentable.

En la sección de "Cultura y política en América Latina" se encuentra un interesante artículo de Eduardo Subirats, denominado "Las poéticas colonizadas de América Latina", que pone al descubierto la ausencia de un proyecto intelectual frente a los dilemas que plantea el siglo XXI.

Desde Estados Unidos y América Latina, el autor formula una dura crítica a los estudios culturales (cultural studies), que diluyen la reflexión civilizatoria, 
la referencia a la realidad ecológica y política global, en una palabra, cualquier proyecto intelectual. Pone en evidencia la fragmentación, departamentalización y compartimentación del conocimiento que hoy están presentes en las instituciones.

Refiere que, en América Latina, los estudios culturales han prosperado en un contexto en el que Estados Unidos ejerce predominio en la esfera económica, mediática y militar. Hoy estamos lejos de los debates intelectuales latinoamericanos en torno al colonialismo y neocolonialismo, la teología de la liberación y las estrategias de resistencia democrática y antiimperialista heredados de una preocupación hermenéutica por el rescate y por la memoria en escritores latinoamericanos de la talla de Juan Rulfo, Augusto Roa Bastos, entre otros, cuya preocupación iba más allá de sus obras literarias y que trazaban un proyecto socialista y democrático.

Los fascismos latinoamericanos de la segunda mitad del siglo XX, señala Subirats, provocaron "una irreversible regresión regional en todos los aspectos de la vida humana". Los golpes militares desmantelaron las organizaciones sociales democráticas y populares, asesinatos masivos de intelectuales y líderes sociales. La crítica del autor va más allá al señalar que al mismo tiempo que se operó la despolitización de los golpes militares, se produjo una expoliación económica y la imposición de una pobreza generalizada en nombre de los derechos jurídicos.

Nos encontramos, según el autor, frente a "un sistema global, de una globalización democrática o de la democracia posmoderna global como nuevo sistema jurídico, financiero y cultural globalmente uniformado". Hoy se descubre una reducción semiótica de toda expresión emocional, compromiso, voluntad de ser y transformarse. El neoliberalismo celebra el fin del intelectual y del arte. En tono irónico, el autor refiere que, a diferencia de los antiguos misioneros, "el misionero global no hostiga a los dioses, ni destruye sus cultos, ni desarraiga las formas de vida que preservaban. Ya hace tiempo que todo eso ha sido reducido a cenizas".
En el presente nos encontramos frente a la reconversión global de proyectos literarios y artísticos que sirven "a la homologación programada de identidades sociales y políticas en las redes semióticas de un desorden mundial sin memorias, sin dioses y sin ser", concluye el autor.

En esta misma sección, encontramos el artículo de Heraclio Bonilla denominado "Los Andes: la metamorfosis y los particularismos de una región". Una de las ideas centrales del autor consiste en mostrar que la región andina, lejos de constituir un espacio homogéneo, como se le ha considerado en varios estudios de las últimas tres décadas, presenta una rica diversidad y por lo mismo resulta necesario un análisis histórico que tome en cuenta los procesos económicos, sociales y políticos de la región, articulados a los ámbitos de nación, clase y etnia. Esto deberá indagarse en cada uno de los espacios nacionales, en el marco de las transformaciones que vienen ocurriendo en el contexto de la mundialización.

El autor plantea que tomar a la región andina como unidad de análisis resulta pertinente toda vez que existe una particular historia, la geografía y el peso de la población indígena. No obstante, advierte el riesgo de una reificación que significa pensar la región como "llave maestra con capacidad de explicar todo lo que ocurra en su contexto", olvidando el tiempo, la historia. Sirve de ejemplo para evidenciar los riesgos de la homogeneización la comparación entre el Perú y el Ecuador que siendo países andinos que comparten todo "desde de su cultura hasta su miseria", han estado dispuestas a enfrentamientos sangrientos.

Los nacionalismos andinos, producto de la historia, "reproducidos y alimentados en la sospecha y en el temor del vecino", se propagan al interior de los territorios nacionales. La paradoja más evidente es la diferencia en el caso del campesinado y sus comunidades, que fueron tomados como "paradigma en la unidad de la región". $\mathrm{El}$ autor advierte que un recorrido del campo andino, 
desde Popayán, en Colombia, hasta Cochabamba, en Bolivia, pone de manifiesto las grandes diferencias: la fortaleza del campesinado en Bolivia y el Perú, frente a la debilidad y dispersión de los campesinos en el Ecuador y en Colombia, resultado del proceso particular de sus respectivas comunidades.

No obstante, el autor refiere que no es lo mismo reconocer la fragmentación territorial y regional que construir el concepto de región, el cual puede ser elaborado de diversas maneras en función de los objetivos particulares. En términos económicos (flujo de bienes y hombres), culturales (normas y valores específicos) o políticos (poder y autoridad que ejerce el grupo dominante). Sin embargo, es la dimensión histórica un elemento central en la definición de una región, la conciencia histórica motiva "la acción de los hombres y que les permite la identificación de sus intereses en su territorio". Esta aproximación es la que hace falta incorporar en las investigaciones que vendrían a enriquecer las visiones de región centradas en las dimensiones económica, política y étnica.

Después de hacer un recorrido histórico por la región y sus particularismos nacionales, el autor llega al final del siglo XX donde se produce un cambio sustancial en el ámbito de lo político, propiciado por la crisis del modelo neoliberal. El relevo político de varios presidentes de corte neoliberal va dando paso a nuevos gobiernos. Pero el derrumbe de las políticas neopopulistas y de la hegemonía del neoliberalismo ha sido posible no por el movimiento obrero y campesino, sino por sectores importantes de la población indígena, de manera quela contradicción clásica entre capital y trabajo fue desplazada por la cultura, que se expresa en las reivindicaciones por la representación y la autonomía. No obstante, Bonilla advierte la necesidad de incorporar la dimensión material del conflicto a fin de no convertir la lucha en un enfrentamiento de signos y símbolos. El autor concluye indicando que hoy, en el umbral del nuevo milenio, "las metamorfosis nacionales y regionales de los Andes" se profundizan y se tejen en el marco de una globalización de nuevo tipo.
En la sección de "Diálogos Latinoamericanos", Emir Sader, Juan Carlos Gómez Leyton y Horacio Tarcus, trazan el itinerario del intelectual chileno Tomás Moulian. Se trata - dice Gómez Leyton- de un intelectual que piensa el socialismo como futuro posible, formado como sociólogo en la Universidad Católica de Chile junto con otros intelectuales como Manuel Antonio Garretón, Joaquín Brunner y Rodrigo Ambrosio, es influenciado por el pensamiento de Althusser y Benedetto Croce. Desde 1967 dicta los primeros cursos y seminarios sobre marxismo, su militancia política en el Movimiento de Acción Popular (MAPU) lo lleva a reforzar su obsesión por el estudio del pensamiento político de Lenin.

En entrevista realizada por Emir Sader resalta su formación profesional y las influencias que recibió del pensamiento leninista y althusseriano, a partir de este último llega a Marx. La Revolución Cubana tuvo gran impacto en su formación política al reafirmar la idea sobre la posibilidad de la revolución en América Latina. Moulian confiesa haber conocido tardíamente el pensamiento de Gramsci, después del golpe militar chileno, lo mismo pasó con la izquierda en el proceso de Unidad Popular.

Uno de las declaraciones interesantes, en su carácter de intelectual del Partido Comunista-MAPU, que estaba en coalición con Allende, era que no esperaba la victoria de la Unidad Popular, de hecho nadie esperaba la victoria. Pero la noticia del triunfo se transformó en una dicotomía: susto y alegría, por la enorme responsabilidad que representaba el triunfo. El golpe y sus consecuencias lo vivió en Chile, su trabajo intelectual, aunque disminuido, lo siguió en la FLACSO.

En ese itinerario intelectual que pasa del triunfo de la Unidad Popular, el golpe de Estado, la derrota de Pinochet y la transición a la democracia, Moulian va externando sus experiencias, sus prácticas. Al final responde a una pregunta clave formulada por Sader en torno a la izquierda actual: piensa que la izquierda chilena se ha neoliberalizado y estatizado, es decir "vive para el poder y no se concibe fuera del 
poder". "Esta izquierda — refiere Moulian — es una izquierda que ha neoliberalizado a la sociedad chilena y al neoliberalizarla en democracia ha aumentado la legitimidad al neoliberalismo".

En su sección de Lecturas Críticas, Marcel Vázquez Castro hace una revisión del libro de Walter Mignolo, La idea de América Latina: la herida colonial y la opción decolonial. Destaca la tesis central de autor que consiste en mostrar que "América" y "América Latina" son ideas inventadas desde la lógica imperialcolonial. En este libro, Mignolo articula lo simbólico con lo político en el presente latinoamericano.

Vázquez destaca en su reseña crítica la idea del pensamiento fronterizo, entendido como un lugar dicotómico de enunciación, históricamente situado en los bordes del sistema mundo moderno-colonial. Se trata de la adaptación de la cosmovisión, conocimientos y memoria de los pueblos indígenas al sistema colonial. De esta manera, la idea de América nació de la articulación entre cosmología cristiana, la economía capitalista y las reacciones decoloniales de los pueblos indígenas. A la idea de pensamiento fronterizo, Vázquez opone el concepto de mestizaje en el que ocurre una interpenetración del pensamiento fronterizo al hegemónico y viceversa, que termina por reconfigurar "tanto las historias locales como los diseños globales".

Para Vázquez, a diferencia del primero, el segundo capítulo del libro de Mignolo es más novedoso: la idea de América Latina como consecuencia de la reconfiguración del mundo moderno-colonial, provocada por el proceso de decolonización del continente americano y emancipación del europeo. Una de las observaciones críticas que hace Vázquez al texto de Mignolo es el hecho de la "confianza ilimitada en los actuales movimientos sociales de pueblos indígenas y afroamericanos", a la que opone la idea de una respuesta hibrida en la que "combina una retórica de afirmación de la identidad de pueblos originarios, instrumentos conceptuales de la izquierda indígena y una práctica que los instala en el terreno de la democracia social republicana".
Finalmente, la episteme de la decolonialidad instala el paradigma de la coexistencia: muchos mundos son posibles. Sin embargo, Vázquez refiere que "es difícil aceptar el quiebre epistemológico” y plantea las siguientes interrogantes: ¿Estas nuevas formas de pensar están realmente fuera del capitalismo global? ¿No estamos convirtiendo a determinados subalternos y a su perspectiva en los nuevos agentes privilegiados del futuro y en la única manera de leer la realidad? ¿Para enfrentarse con éxito a las formas de dominación del capitalismo global es necesario desvincular el marxismo del proyecto decolonial? ¿Cuál sería el diálogo posible entre los movimientos de emancipación que surgen en el centro del sistema-mundo y el proyecto decolonial?

Finalmente, en la sección de Documentos aparece el texto del discurso del presidente de Ecuador, Rafael Correa, con motivo al $50^{\circ}$ aniversario de la Facultad Latinoamericana de Ciencias Sociales (FLACSO). Se trata de un texto muy interesante que refleja la visión de un mandatario latinoamericano en la búsqueda de opciones distintas en el campo de las ciencias sociales y su vínculo con un compromiso por la transformación de la realidad latinoamericana. En palabras de Correa, es una invitación "a construir una academia comprometida con las necesidades de América Latina y atenta a los procesos de cambio que hoy por hoy estamos experimentando".

Correa refiere a la historia, al contexto que dio vida a FLACSO. La Guerra Fría, la Revolución Cubana, los movimientos guerrilleros y la Teología de la Liberación, sirven de marco al surgimiento de la FLACSO. Recuerda que una de las líneas de investigación que se privilegiaron en su momento fue el tema del desarrollo en una perspectiva articulada con lo económico, social, cultural y político. También la educación fue abordada como un campo del desarrollo, con la crítica de la teoría del capital humano se establecen conexiones entre la formación de los recursos humanos y el crecimiento económico. En todos los estudios la historia ocupa un lugar de primer orden en la comprensión de la especificidad de la realidad latinoamericana. 
Correa no soslaya que en el presente existe una crisis en el pensamiento latinoamericano y formula dos preguntas fundamentales: ¿Qué es lo que en rigor justifica la existencia y desarrollo de las ciencias sociales? ¿Cuál es la misión que como científico social le corresponde a cada uno de nosotros ante la dinámica, contradictoria y en muchos aspectos dolorosa de la realidad del mundo contemporánea? La respuesta a estas interrogantes es directa: contribuir a la comprensión de los fenómenos del mundo social y aumentar nuestra capacidad de actuar en la construcción de una sociedad mejor. Esto lleva a una doble reflexión: en torno al diálogo de saberes que permiten incrementar la capacidad comprensiva, y de la distinción entre objetividad y neutralidad. Correa es muy enfático en señalar que no debe confundirse estos dos términos pues debemos ser científicos sociales objetivos pero no neutros.

El presidente Correa formula una serie de preguntas vinculadas con el quehacer investigativo que hoy tiene un valor fundamental en la construcción de una agenda frente a los retos que plantea el siglo XXI: ¿De dónde surgen los temas de investigación en las ciencias sociales actuales? ¿Desde qué lugar se construyen las preguntas de investigación? ¿Cuál es la economía política de las teorías dominantes en las ciencias sociales?, en última instancia ¿Cuál es la forma de producción del conocimiento que parece funcionar en la academia de América Latina? Correa está convencido de que los espacios académicos son espacios de disputa ideológica que se orientan a la construcción de hegemonía de unos intereses por sobre otros, de unas visiones del mundo por otras y pone como ejemplo cómo la academia latinoamericana fue colonizada por teorías y recetas metodológicas que surgieron en los países centrales: la economía positivista en el campo de la ciencia social, la racional choice en el ámbito de la ciencia política y en la sociología política, son ejemplos del colonialismo intelectual en América Latina, cuestión que impide ver otras opciones de construir los problemas de investigación y los objetos de estudio relevantes para los proyectos de cambio.

En resumen, este primer número de la Revista Crítica y Emancipación reúne diversos materiales que en conjunto permiten descubrir esfuerzos renovados por construir una nueva epistemología para pensar la región latinoamericana y contribuir a su transformación en un contexto de pluralidad pero con una visión desde abajo, que rebase la perspectiva de los países centrales que hasta ahora no ha logrado contribuir a superar las grandes desigualdades y la pobreza de los sectores mayoritarios de la población.

Daniel Villafuerte Solís

Integrante del Cuerpo Académico Política, Diferencia y Fronteras CESMECA-UNICACH. 